\title{
Spin Polarized Transport through Quantum Dots: Coulomb Blockade and Kondo Effect
}

\author{
J. BARNAŚ ${ }^{a, b}$, R. ŚWIRKOWICZ ${ }^{c}$, M. WILCZYŃSKI ${ }^{c}$, \\ I. Weymann ${ }^{a}$, J. Martinek ${ }^{b, d}$ And V. DugaeV ${ }^{e}$ \\ ${ }^{a}$ Depart. of Physics, A. Mickiewicz University \\ Umultowska 85, 61-614 Poznań, Poland \\ ${ }^{b}$ Institute of Molecular Physics, Polish Academy of Sciences \\ Smoluchowskiego 17, 60-179 Poznań, Poland \\ ${ }^{c}$ Faculty of Physics, Warsaw Technical University \\ Koszykowa 75, 00-662 Warszawa, Poland \\ ${ }^{d}$ Institut für Theoretische Physik, Universität Karlsruhe \\ 76128 Karlsruhe, Germany \\ ${ }^{e}$ Max-Planck-Institut für Mikrostrukturphysik \\ Weinberg 2, 06120 Halle, Germany
}

Spin related effects in electronic transport through quantum dots, coupled via tunneling barriers to two metallic leads, are discussed from the point of view of fundamental physics and possible applications in spin electronics. The effects follow either from long spin relaxation time in the dots or from spin dependent tunneling through the barriers when the external leads are ferromagnetic. In the former case large nonequilibrium spin fluctuations in the dot can be induced by flowing current. These fluctuations modify transport characteristics, particularly the shape of the Coulomb steps. In the latter case electric current depends on magnetic configuration of the system, and tunnel magnetoresistance effect due to magnetization rotation can occur. Transport properties in the weak coupling regime are described perturbatively in the first (sequential) and second (cotunneling) orders. In the strong coupling regime, on the other hand, the equation of motion for nonequilibrium Green functions is used to calculate electric current at low temperatures, where the Kondo peak in conductance is formed in the zero bias regime. In symmetrical systems the Kondo peak is split in the parallel magnetic configuration, whereas no splitting occurs for the antiparallel alignment. Theoretical results are discussed in view of a vailable experimental data.

PACS numbers: 72.25.-b, 73.23.Hk, 72.15.Qm 


\section{Introduction}

More than a decade ago Grünberg et al. [1] discovered antiferromagnetic interlayer exchange coupling in artificially layered structures consisting of two $3 d$ ferromagnetic metallic films separated by a nonmagnetic metallic spacer. It turned out later that the sign of the corresponding exchange parameter oscillates with the spacer thickness and disappears when the thickness exceeds several tens of angstroms [2]. A direct consequence of the presence of antiferromagnetic interlayer coupling was the subsequent discovery of the giant magnetoresistance (GMR) effect $[3,4]$. This discovery initiated broad interest in spin polarized transport in artificial and natural nanoscopic systems.

A typical structure, in which the GMR effect occurs, is a multilayer in which adjacent ferromagnetic films are coupled antiferromagnetically across a nonmagnetic spacer, so they have antiparallel magnetizations in zero magnetic field. When an external magnetic field is applied, the magnetizations rotate from antiparallel configuration to parallel one, and this rotation is associated with a decrease in electrical resistance of the system (an increase is also possible [5]). The effect was found for electric current flowing in the film plane [3, 4], as well as for current flowing perpendicularly to the film plane [6].

To observe the GMR effect one needs to create the antiparallel alignment of the film magnetizations. In early experiments such an alignment was a consequence of antiferromagnetic interlayer exchange coupling. However, the exchange coupling is not a necessary condition and its presence is even not advisable from the application point of view because it enhances the saturation field and diminishes field sensitivity of the effect. Fortunately, the antiparallel alignment can also be obtained by other means. If, for instance, the two magnetic films in a trilayer structure have different coercive fields, then there is a range of magnetic fields during scanning through the hysteresis loop, where the magnetizations are antiparallel [7]. A specific example of such structures are spin valves, where one magnetic film is pinned by exchange anisotropy to an antiferromagnetic substrate, whereas the other one is magnetically free.

The GMR effect is described quantitatively by the ratio $\Delta R / R_{\uparrow \uparrow}=\left(R_{\uparrow \downarrow}-\right.$ $\left.R_{\uparrow \uparrow}\right) / R_{\uparrow \uparrow}$, where $R_{\uparrow \downarrow}$ and $R_{\uparrow \uparrow}$ are the total resistances of a multilayer in the antiparallel and parallel magnetic configurations, respectively. A typical value of this factor is of an order of $0.1 \div 1.0$. In the case of the GMR effect described above, all components of a multilayer are metallic. Electrical resistivity of such structures is therefore typical of metallic systems. However, a similar effect also occurs when the metallic spacer layer in a trilayer structure is replaced by an insulating barrier $[8,9]$. Vertical transport occurs then owing to electron tunneling through the barriers. Consequently, a typical conductance is then several orders of magnitude smaller than for metallic spacers. The corresponding magnetoresistance effect is known as tunnel magnetorsistance (TMR). 
The TMR effect can exist in simple planar junctions as well as in more complex ones, e.g., in double-barrier junctions [10]. An example of the latter systems are junctions with the central electrode (the part between the two barriers, called an island in the following) being small enough, so that the effects due to charging of the electrode with single electrons are visible in the corresponding current-voltage characteristics. In the case of nonmagnetic systems, and when discreteness of the density of states of the island can be neglected, this leads to the well-known Coulomb blockade of electric current below a certain threshold voltage and to characteristic Coulomb steps. These features disappear when the thermal energy $k_{\mathrm{B}} T$ exceeds the charging energy $E_{\mathrm{c}}$. When the system is in a blocked state, the Coulomb blockade can be removed by applying a gate voltage $V_{\mathrm{g}}$ to the central electrode. The system acts then like a transistor, in which electrons are transmitted in a correlated way one by one, and therefore the device is called single-electron transistor (SET) [11]. The interplay of magnetic properties of ferromagnetic junctions and charging effects was studied only very recently [12-14].

Additional features in transport characteristics arise from size quantization of electron states in the central electrode $[15,16]$. When the island is a semiconducting quantum dot or a single molecule, or an ultra-small metallic particle, separation between discrete states can be comparable or even larger than the charging energy, and also much larger than thermal energy. In this paper we will describe just the interplay of the charging effects, discreteness of the density of states, and magnetic properties. Accordingly, we consider a quantum dot connected to two external electrodes which can be magnetic in a general case. We will describe the limit of weak coupling between the dot and electrodes, when the Coulomb blockade and other charging phenomena dominate, as well as the strong coupling limit, where the Kondo effect may be observed at low temperatures.

The spin effects in electronic transport, discussed in this paper, are due to either a difference in relevant time scales for spin and charge degrees of freedom or spin polarization of electric current when the external and/or central electrodes are ferromagnetic. Generally, one can distinguish three relevant time scales in electronic transport through ferromagnetic or nonmagnetic quantum dots: (i) spin-conserving energy relaxation time on the dot $\tau_{\epsilon}$, (ii) current injection time $\tau_{\mathrm{I}}$, and (iii) spin-flip relaxation time $\tau_{\mathrm{sf}}$. In real systems $\tau_{\epsilon}$ is usually the shortest time scale, $\tau_{\epsilon}<10^{-9} \mathrm{~s}$, whereas $\tau_{\mathrm{sf}}$ can be in the range of $10^{-9} \div 10^{-6} \mathrm{~s}$. On the other hand, for the current of $1 \mathrm{nA}$, the injection time is of the order of $10^{-9} \mathrm{~s}$. Accordingly, one may assume that the shortest time scale in the systems under consideration is the spin-conserving energy relaxation time, while the longest one is the spin-flip relaxation time, $\tau_{\mathrm{sf}} \gg \tau_{\mathrm{I}} \gg \tau_{\epsilon}$ (slow spin relaxation limit). In other words, one may assume that after tunneling to the island, the electron relaxes energetically to the relevant Fermi level before next tunneling processes take place, whereas electron spin preserves its orientation for a time much longer than the 
time between successive tunneling events (injection time). In the case of magnetic systems this results in a spin splitting of the steady-state Fermi level of the island. Apart from this, one may expect large nonequilibrium spin fluctuations on the island, even if the system is entirely nonmagnetic. These large spin fluctuations may lead to observable effects in electronic transport [17, 18].

In some systems, however, the conditions of slow spin relaxation are not fulfilled and $\tau_{\text {sf }}$ becomes of the order of $\tau_{\mathrm{I}}$ or even shorter. In this so-called fast spin relaxation limit, the spin effects due to different time scales disappear. However, the effects due to spin dependent tunneling across the barriers can still occur when some components of the system are ferromagnetic.

When the quantum dot contains an odd number of electrons, the Kondo effect in electronic transport through the dot can be observed at low temperatures $[19,20]$. The effect is fundamentally similar to the Kondo effect observed in alloys with magnetic impurities. However, there is an important difference - the Kondo effect in alloys gives rise to a minimum in resistivity below the Kondo temperature, while in the case of transport through quantum dots it gives rise to a minimum in conductance. Spin polarization of conduction electrons in ferromagnetic electrodes leads to new features of the Kondo phenomenon [21-23].

In Sect. 2 we discuss spin effects in quantum dots connected to nonmagnetic leads. Such spin effects follow either from exchange coupling between electrons on the dot or from a difference between energy and spin relaxation times. In Sect. 3 we consider spin polarized transport through a quantum dot in the sequential tunneling and cotunneling regimes. Finally, in Sect. 4 we discuss spin polarized transport through quantum dots in the Kondo regime. Possible applications in spintronics are discussed in Sect. 5, where also concluding remarks are given.

\section{Spin effects in quantum dots coupled to nonmagnetic leads}

Exchange interaction between electrons in quantum dots leads to effects which are similar to those occurring in natural atoms. One of its consequences is lifting the spin degeneracy of discrete levels. Moreover, in some cases consecutive discrete levels may become populated following Hund's rule. Accordingly, the dot can acquire a stable magnetic moment, similar to the magnetic moment of certain atoms. Hund's rule may then lead to spin blockade phenomenon in electronic transport through the dot. In other words, electrons with one spin orientation can tunnel from the source electrode to the dot and then from the dot to the drain electrode, whereas tunneling of electrons with opposite spin orientation is suppressed by Hund's rule [24].

There are also other spin effects in electronic transport, which are due to nonequilibrium spin fluctuations in quantum dots. Such spin fluctuations occur when spin-flip relaxation time $\tau_{\mathrm{sf}}$ is long compared to other relaxation processes like the energy relaxation time $\tau_{\epsilon}$ and the injection time $\tau_{\mathrm{I}}$. These effects occur 
in the nonlinear current-voltage regime, and when the size effects play a role. Moreover, they can occur even in the absence of exchange interactions on the dot. In the following part of this section we will consider in more details the role of nonequilibrium spin fluctuations in transport processes.

Assume that a quantum dot is connected to two nonmagnetic leads. When positive bias corresponds to electrons flowing from right to left, then the electric current $I$ flowing through the dot is determined by the following formula:

$$
I=I_{\mathrm{r}}=e \sum_{\sigma} \sum_{N_{\uparrow}, N_{\downarrow}} P\left(N_{\uparrow}, N_{\downarrow}\right)\left\{\Gamma_{r \sigma}^{+}\left(N_{\uparrow}, N_{\downarrow}\right)-\Gamma_{r \sigma}^{-}\left(N_{\uparrow}, N_{\downarrow}\right)\right\}
$$

where $-e$ is the electron charge $(e>0), P\left(N_{\uparrow}, N_{\downarrow}\right)$ is the probability to have on the $\operatorname{dot} N_{\uparrow}$ electrons with spin $\uparrow$ and $N_{\downarrow}$ electrons with spin $\downarrow$, whereas $\Gamma_{r \sigma}^{ \pm}\left(N_{\uparrow}, N_{\downarrow}\right)$ is the tunneling probability to $(+)$ and off $(-)$ the dot through the right barrier. Similar probability may be defined for tunneling through the left barrier ( $I=$ $I_{1}=I_{\mathrm{r}}$ ). In the simplest "orthodox" tunneling theory, these probabilities can be calculated from the Fermi golden rule. On the other hand, the probability $P\left(N_{\uparrow}, N_{\downarrow}\right)$ can be determined from the relevant master equation, which takes into account a detailed balance of electrons tunneling to and off the dot $[16,17]$.

A state of the dot can be characterized either by the numbers $N_{\uparrow}$ and $N_{\downarrow}$, or equivalently by the numbers $N=N_{\uparrow}+N_{\downarrow}$ (charge state) and $M=N_{\uparrow}-N_{\downarrow}$ (magnetic state). When the spin relaxation is slow, large spin fluctuations on the dot can arise in a nonequilibrium situation (biased system). In Fig. 1 two different magnetic states are shown schematically assuming the constant level spacing $\Delta E$. These different magnetic states correspond to the same charge state. Owing to

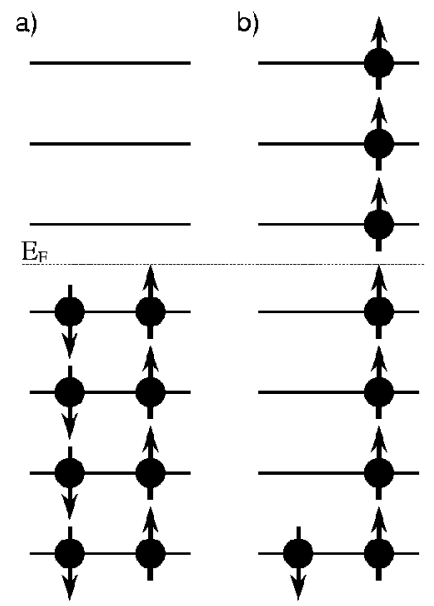

Fig. 1. Two magnetic states of the dot, which correspond to the same charge state. The state (a) corresponds to short spin-flip relaxation time, whereas the state (b) is relevant when the spin-flip relaxation time is long. 
the discrete energy structure, a transition from the $N$ to $N+1$ charge states can occur at a bias lower than in the absence of spin fluctuations. This transition involves high-spin states (states corresponding to large values of $M$ ). Consequently, the transition between different charge states is smoother and the Coulomb steps in the current-voltage characteristics become less pronounced. The effect is relevant as long as the energy of thermal fluctuations is lower than the energy of nonequilibrium spin fluctuations. Thus, even if the discrete states are not resolved $\left(k_{\mathrm{B}} T \gtrsim \Delta E\right)$, the spin fluctuations can lead to observable effects.

The role of nonequilibrium spin fluctuations in electronic transport through quantum dots is shown schematically in Fig. 2, where positions of the conductance peaks (current steps) are indicated in the low-temperature limit, $k_{\mathrm{B}} T \ll \Delta E$. The regions marked with $N, N+1$, and $N+2$ are the Coulomb blockade ones and correspond to $N, N+1$, and $N+2$ electrons on the dot, respectively. In the fast spin relaxation limit, the solid lines determine the well-known Coulomb blockade diamond structure. The regions denoted as SETT (single electron tunneling transport) and DETT (double electron tunneling transport) correspond to the cases where one and two excess electrons can occur in the dot, respectively, when appropriate gate and transport voltages are applied. The dotted lines indicate standard resonance peaks following from the discrete structure. When spin relaxation is slow, there are new resonance peaks indicated by the dashed lines which start inside the SETT diamond when a particular spin excitation appears. Due to nonequilibrium spin fluctuations, the double electron tunneling transport can occur already within the SETT diamond region (the regions marked in gray in Fig. 2).

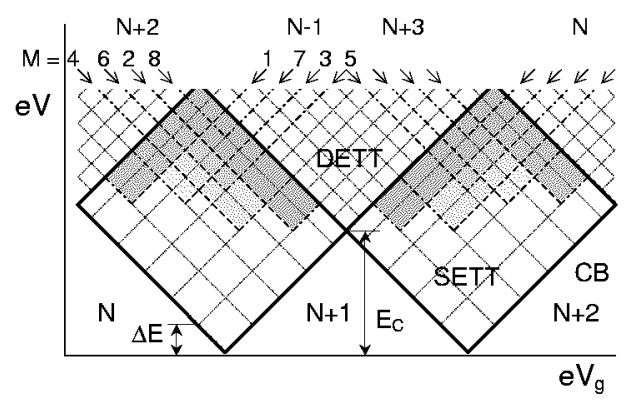

Fig. 2. The scheme of conductance peaks in the $V-V_{\mathrm{g}}$ plane for a symmetric junction; $E_{\mathrm{C}} / \Delta E=4.5$ and $k_{\mathrm{B}} T \ll \Delta E$. Solid lines determine the Coulomb blockade diamond structure indicating also the onset of single electron tunneling transport (SETT) and double electron tunneling transport (DETT) in the absence of nonequilibrium spin fluctuations. Dotted lines are usual effects related to the offset in transport of new discrete energy levels. Dashed and dotted-dashed lines indicate the onset of the consecutive charge states due to particular spin excitations (as indicated). 
Recently, Fujisawa et al. [18] obtained experimental results on small vertical QDs at low temperatures, which confirm the role of nonequilibrium spin fluctuations in electronic transport. One should also note that the borders between SETT and DETT diamonds observed in earlier experiments have features indicating the role of nonequilibrium spin fluctuations, too.

\section{Spin polarized transport through quantum dots in the sequential tunneling and cotunneling regimes}

When the quantum dot is attached to ferromagnetic electrodes, the leading spin effects originate from spin polarization of injected electrons. If the spin relaxation time on the dot is sufficiently long, spin dependent tunneling through the barriers gives rise to the TMR effect. The relevant experiments have been performed with carbon nanotubes (CNTs) of finite length used as the central electrode [25]. When CNT is not too long, it behaves like a quantum dot with discrete energy levels and with a finite number of electrons. Moreover, the corresponding spin relaxation time can be relatively long. Indeed, spin polarized transport through CNT attached to two Co electrodes revealed long spin coherence length, which gave rise to large TMR effect [25].

To describe the main features of spin polarized electronic transport through quantum dots, let us consider the limiting case of a single-level dot. Electron correlations on the dot can be then taken into account in the Hubbard form and the Hamiltonian of the whole system can be written as

$$
\begin{aligned}
H= & \sum_{\alpha=1, \mathrm{r}} \sum_{k \sigma} \varepsilon_{\alpha k \sigma} c_{\alpha k \sigma}^{\dagger} c_{\alpha k \sigma}+\sum_{\sigma} \epsilon_{\mathrm{d}} d_{\sigma}^{\dagger} d_{\sigma}+U d_{\uparrow}^{\dagger} d_{\uparrow} d_{\downarrow}^{\dagger} d_{\downarrow} \\
& +\sum_{\alpha=1, \mathrm{r}} \sum_{k \sigma}\left(V_{\alpha k \sigma} c_{\alpha k \sigma}^{\dagger} d_{\sigma}+V_{\alpha k \sigma}^{*} d_{\sigma}^{\dagger} c_{\alpha k \sigma}\right),
\end{aligned}
$$

where the first term on the right hand side describes the two electrodes in the noninteracting quasi-particle approximation, the second and third terms describe the dot with $\epsilon_{\mathrm{d}}$ being the energy of the discrete level and $U$ denoting the electron correlation parameter. The last term takes into account tunneling processes between the dot and the leads, with the spin dependent coupling parameters $V_{\alpha k \sigma}$. The energy level $\epsilon_{\mathrm{d}}$ includes the electrostatic part, $\epsilon_{\mathrm{d}}=\epsilon_{\mathrm{d}}^{0}-e U_{\mathrm{e}}$, where $\epsilon_{\mathrm{d}}^{0}$ is the level energy at zero bias, and $U_{\mathrm{e}}$ is the electrostatic potential of the dot. Similarly, the single-electron energy of the leads includes the corresponding electrostatic energy, too.

Strength of the coupling between electrodes and the dot can be described by $\Gamma_{\alpha \sigma}(E)=2 \pi \sum_{k}\left|V_{\alpha k \sigma}\right|^{2} \delta\left(E-\epsilon_{\alpha k \sigma}\right)$, for $\alpha=1$, r. In the following we consider only colinear magnetic configurations, i.e., the parallel and antiparallel orientations of magnetic moments of the leads. When the tunneling rates are small (small values of $\Gamma_{\mathrm{r}(1) \sigma}(E)$ ), transport characteristics can be calculated within the master equation 
technique, with the tunneling rates calculated in the lowest order from the Fermi golden rule. However, such a description does not properly take into account the electron correlations on the dot. It also is not applicable in the strong tunneling regime and at low temperatures, where many body effects become important and lead to the Kondo effect. However, when transport is dominated by sequential tunneling processes, such a description gives reasonable results [26, 27].

The description based on sequential tunneling breaks down in the Coulomb blockade regime, where sequential tunneling is suppressed by the Coulomb energy. Electric transport in this regime is still possible via higher-order tunneling processes. The dominant processes are the ones of the second order, known also as cotunneling, where two tunneling events through the barriers occur simultaneously, so the charge state of the dot is not changed. For weak coupling between the dot and electrodes, the sequential and cotunneling processes can be calculated perturbatively, and in a simplified description one can use Born approximation to calculate the appropriate tunneling rates. However, the Born approximation is valid only at small bias voltage. When the bias is close to the threshold voltage, one has to use a more accurate description, e.g., by summing a certain class of higher order terms to remove divergences. The most suitable method seems to be the real time diagrammatic technique, in which the density matrix is expanded into a series with respect to tunneling Hamiltonian, and each tunneling event corresponds to a certain class of diagrams [28]. This technique allows a direct control of tunneling events taken into considerations.

Let us assume that the discrete level of the dot is below the Fermi level of the leads in equilibrium and the correlation parameter $U$ is sufficiently large so the dot in equilibrium is occupied by a single electron only. In the limit of small bias, the sequential tunneling processes are exponentially suppressed and only the following elastic cotunneling processes can occur: (i) an electron with spin opposite to that on the dot enters the dot and then one electron leaves the dot, and (ii) an electron leaves the dot and another electron enters the dot. When the spin state of the dot is not changed, such elastic tunneling processes are coherent. When, however, the spin state is changed, the tunneling processes destroy coherence. In any case, one can have one-barrier and/or two-barrier cotunneling processes. The former processes contribute directly to electric current. The latter ones, on the other hand, do not transport electron charge, so they do not contribute directly to the flowing current. However, they can contribute indirectly by modifying magnetic state of the dot. In addition, there are also other second order tunneling processes which effectively lead to renormalization of the sequential tunneling. Thus, the total second order contribution to tunneling current can be positive as well as negative.

In Fig. 3 we show the TMR in the Coulumb blockade regime, when only sequential tunneling processes are taken into account and also when cotunneling events are included. It is evident that cotunneling processes enhance the TMR 


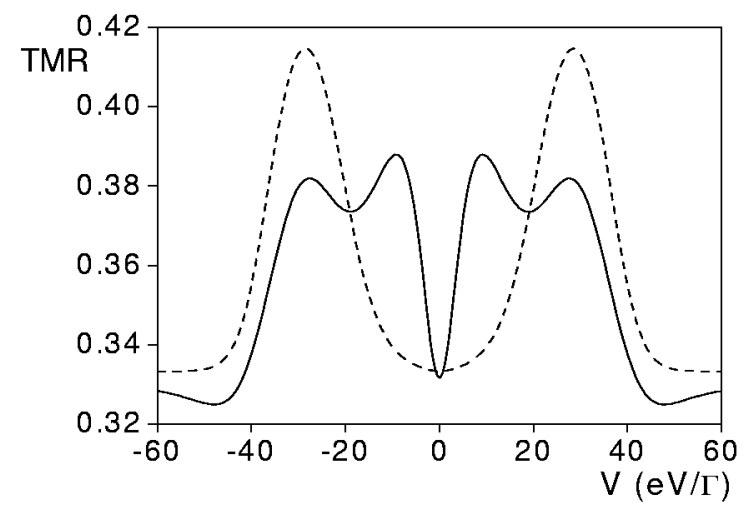

Fig. 3. TMR as a function of bias voltage, calculated for a symmetrical system in the sequential tunneling limit (dashed line) and with the cotunneling processes included (solid line). The parameters are: $k_{\mathrm{B}} T=2 \Gamma, \epsilon_{\mathrm{d}}^{0}=-10 \Gamma, U=30 \Gamma$, polarization of the leads $P=0.5$ and $\Gamma=\Gamma_{\sigma}+\Gamma_{-\sigma}$, with $\Gamma_{\sigma}=\Gamma_{\mathrm{l} \sigma}=\Gamma_{\mathrm{r} \sigma}$ in the parallel configuration.

effect in the Coulomb blockade regime. Such an enhancement was also observed in the case of electron tunneling through small metallic magnetic grains. Another interesting effect is the suppression of TMR obtained in the sequential tunneling limit close to the resonance. This follows from suppression of electric current by renormalization of the sequential tunneling due to the second order tunneling processes.

\section{Kondo effect}

The Kondo phenomenon in electronic transport through quantum dots strongly coupled to metallic nonmagnetic leads was predicted theoretically long time ago [19]. The prediction was recently confirmed experimentally [20]. There is some difference between the Kondo effect in quantum dots and that observed in alloys with magnetic impurities. In the latter case there is a minimum in resistivity at a certain temperature (known as the Kondo temperature), whereas in the former one there is a minimum in conductance. However, the physical mechanism leading to the anomalies is similar in both cases and is based on the formation of Kondo peak in the density of states at the Fermi level. This Kondo peak enhances transmission through quantum dots leading to almost perfect transmission at zero temperature, i.e., to the conductance $2 e^{2} / h$.

Several theoretical techniques have been developed in the past decade to study the Kondo phenomenon in quantum dots. The limit of linear response (limit of zero bias voltage) can be treated by equilibrium methods. However, the problem becomes more complex when a bias voltage is applied, which drives the system out of equilibrium. It was shown that the Kondo peak becomes then split and the two peaks are formed at the Fermi levels of both leads attached to the dot [29]. 
It is only very recently when the problem of the Kondo effect in quantum dots attached to ferromagnetic leads was addressed [21-23]. There are no relevant experimental data yet, but the existing theoretical results clearly indicate that the Kondo effect can be observed, although the predictions differ in certain aspects. The key difference concerns splitting of the Kondo peak in the density of states at equilibrium. Such a splitting was predicted in Ref. [22], where the Kondo effect in the large $U$ limit was analyzed within the equation of motion method, and also by some scaling considerations.

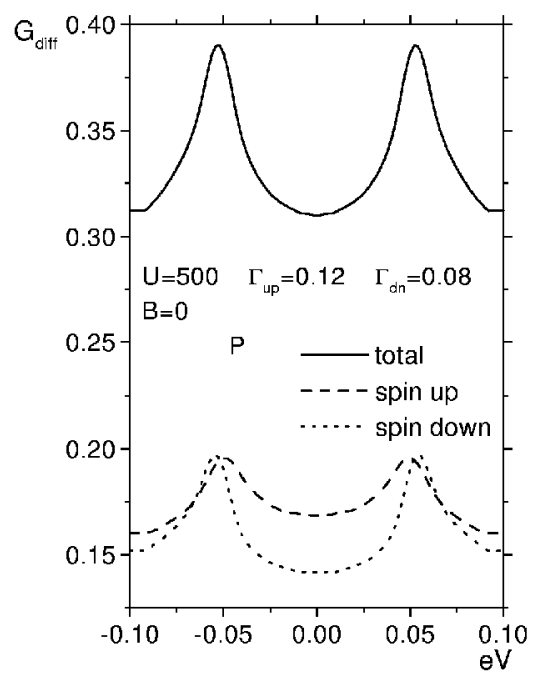

Fig. 4. Differential conductance in the Kondo regime, calculated for zero magnetic field $B$ and for the parallel configuration. The energy parameters are given in dimensionless units (the unit is $1 / 50$ of the electron band width). Apart from this, $\Gamma_{\mathrm{up}}=\Gamma_{1 \uparrow}=\Gamma_{\mathrm{r}}$ and $\Gamma_{\mathrm{dn}}=\Gamma_{1 \downarrow}=\Gamma_{\mathrm{r} \downarrow}$.

One of the methods applicable to systems out of equilibrium is the nonequilibrium Green function technique. To calculate density of states and electric current one needs both retarded and lesser (correlation) Green functions. In Ref. [23] the equation of motion was used to calculate both Green functions on equal footing, i.e., within the same approximation scheme. Assuming the model Hamiltonian given by Eq. (2), the following formula for electric current was derived there:

$$
\begin{aligned}
I= & \frac{\mathrm{i} e}{2 h} \sum_{\sigma} \int \frac{\mathrm{d} E}{2 \pi} \frac{\Gamma_{\mathrm{l} \sigma}(E) \tilde{\Gamma}_{\mathrm{r} \sigma}(E)+\Gamma_{\mathrm{r} \sigma}(E) \widetilde{\Gamma}_{1 \sigma}(E)}{\widetilde{\Gamma}_{1 \sigma}(E)+\widetilde{\Gamma}_{\mathrm{r} \sigma}(E)} \\
& \times\left[G_{\sigma \sigma}^{\mathrm{r}}(E)-G_{\sigma \sigma}^{\mathrm{a}}(E)\right]\left[f_{\mathrm{l}}(E)-f_{\mathrm{r}}(E)\right]
\end{aligned}
$$

which is valid for collinear magnetic configurations. Here, $f_{\alpha}(E)$ is the Fermi distribution function in the electrode $\alpha, G_{\sigma \sigma}^{\mathrm{r}(\mathrm{a})}$ is retarded (advanced) Green's function of the dot, and $\widetilde{\Gamma}_{\alpha \sigma}(E)$ are certain effective coupling parameters which are closely 
related to $\Gamma_{\alpha \sigma}(E)$ [23]. The Green functions depend on the occupation number $n_{\sigma}=\left\langle d_{\sigma}^{+} d_{\sigma}\right\rangle$, which can be determined from the formula

$$
n_{\sigma}=\int \frac{\mathrm{d} E}{2 \pi} \frac{\widetilde{\Gamma}_{\mathrm{l} \sigma}(E) f_{\mathrm{l}}(E)+\widetilde{\Gamma}_{\mathrm{r} \sigma}(E) f_{\mathrm{r}}(E)}{\widetilde{\Gamma}_{\mathrm{l} \sigma}(E)+\widetilde{\Gamma}_{\mathrm{r} \sigma}(E)}\left[G_{\sigma \sigma}^{\mathrm{r}}(E)-G_{\sigma \sigma}^{\mathrm{a}}(E)\right]
$$

All information on the Kondo anomaly in the density of states is contained in the Green functions. In the case of nonmagnetic systems, the Kondo peak in the density of states gives rise to a Kondo peak in the differential conductance $G_{\text {diff }}$, which occurs in the zero bias regime. When the dot is symmetrically coupled to two ferromagnetic leads, a splitting of the Kondo peak in the density of states occurs in the parallel configuration. This, in turn, leads to splitting of the Kondo peak in the differential conductance. This is shown in Fig. 4, where $G_{\text {diff }}$ is presented as a function of the bias voltage. It is worth noting that by applying a magnetic field one can compensate the splitting and restore the full strong coupling Kondo effect. In the case of antiparallel configuration, there is no splitting of the Kondo peak in the density of states and no splitting of the zero-bias anomaly in the differential conductance. The situation is then similar to what one expects in quantum dots coupled to nonmagnetic leads. This behavior of differential conductance leads to negative TMR in the small bias range.

\section{Possible applications and concluding remarks}

For practical applications in spin electronics, the devices based on quantum dots should operate at temperatures which are much higher than the Kondo temperature [30]. One kind of possible applications of quantum dots are spin filters. We already mentioned before that quantum dots with energy states obeying Hund's rule may be used as spin filters. From similar reasons one can use magnetic quantum dots as spin filters, too. When the spin splitting of the dot level is sufficiently large, one can reach the spin blockade regime for electronic transport, similar to the spin blockade due to Hund's rule.

Another interesting application of quantum dots is a spin diode. In such a device, a quantum dot transmits electric current for one bias polarization and blocks the current for the opposite bias. An example of such a system is a quantum dot coupled to two ferromagnetic electrodes, one of them being half metallic with almost full spin polarization of electron states at the Fermi level.

Since the devices should operate at high temperatures, the formal description of the transport characteristics may be simplified. In the framework of the equation of motion method one can limit oneself to the Hartree-Fock approximation when deriving the Green functions. In that case the formula for electric current becomes simplified and has the form of Eq. (3), but with $\widetilde{\Gamma}_{\alpha \sigma}(E)$ replaced by $\Gamma_{\alpha \sigma}(E)[31,32]$.

Let us consider qualitatively the case where the right electrode is ferromagnetic (it can be also nonmagnetic), whereas the left one is half-metallic. Assume 
that the discrete level $\epsilon_{\mathrm{d}}^{0}$ is well below the Fermi level of the electrodes at equilibrium, and $\epsilon_{\mathrm{d}}^{0}+U$ is slightly above the Fermi level. When $\epsilon_{\mathrm{d}}^{0}+U$ is much larger than the thermal energy, the discrete level is always occupied with a single electron in equilibrium. For positive bias the current starts to flow when $\epsilon_{\mathrm{d}}+U$ crosses the Fermi level of the source electrode. The situation is significantly different for negative bias. When $\epsilon_{\mathrm{d}}+U$ crosses the Fermi level of the source electrode, only a small current flows due to cotunneling processes, while sequential tunneling is suppressed. This is because an electron with spin parallel to the spin of electrons in the half-metallic source electrode blocks sequential tunneling processes.

In conclusion, one may state that quantum dots offer new physics and also possibilities of new devices for applications in nanoelectronics and spin electronics.

\section{Acknowledgments}

This work was supported by the State Committee for Scientific Research through the project PBZ-KBN-044/P03-2001.

\section{References}

[1] P. Grünberg, R. Schreiber, Y. Pang, M.B. Brodsky, H. Sowers, Phys. Rev. Lett. 57, $2442(1986)$.

[2] S.S.P. Parkin, N. More, K.P. Roche, Phys. Rev. Lett. 44, 2104 (1990).

[3] M.N. Baibich, J.M. Broto, A. Fert, F. Nguyen van Dau, F. Petroff, P. Etienne, G. Creuzet, A. Friederich, J. Chazelas, Phys. Rev. Lett. 61, 5472 (1988).

[4] G. Binasch, P. Grünberg, F. Saurenbach, W. Zinn, Phys. Rev. B 39, 4818 (1989).

[5] J.M. George, L.G. Pereira, A. Barthelemy, F. Petroff, L. Steren, J.L. Duvail, A. Fero, R. Loloee, P. Holody, P.A. Schroeder, Phys. Rev. Lett. 42, 308 (1994).

[6] M.A.M. Gijs, S.K.J. Lenczowski, J.B. Giesbers, Phys. Rev. Lett. 40, 7343 (1993).

[7] J. Barnaś, A. Fuss, R.E. Camley, P. Grünberg, W. Zinn, Phys. Rev. B 42, 8110 (1990).

[8] M. Julliere, Phys. Lett. A 54, 225 (1975).

[9] J.S. Moodera, L.R. Kinder, T.M. Wong, R. Meservey, Phys. Rev. Lett. 74, 3273 (1995).

[10] L.F. Schelp, A. Fert, F. Fettar, P. Holody, S.F. Lee, J.L. Maurice, F. Petrofr, A. Vaures, Phys. Rev. B 56, R5647 (1997); M. Wilczyński, J. Barnaś, J. Magn. Magn. Mater. 221, 373 (2000).

[11] For a review see Single Charge Tunneling, Eds. H. Grabert, M.H. Devoret, NATO ASI Series, Vol. 294, Plenum Press, New York 1992.

[12] K. Ono, H. Shimada, S. Kozayashi, Y. Outuka, J. Phys. Soc. Jpn. 69, 3959 (1996); H. Shimada, K. Ono, Y. Outuka, J. Phys. Soc. Jpn. 67, 1359 (1998).

[13] J. Barnaś, A. Fert, Phys. Rev. Lett. 80, 1088 (1998); Europhys. Lett. 24, 85 (1998).

[14] S. Takahashi, S. Maekawa, Phys. Rev. Lett. 80, 1758 (1998). 
[15] D.C. Ralph, S. Gueron, C.T. Black, M. Tinkham, Physica B 280, 420 (2000).

[16] J. Barnaś, J. Martinek, G. Michałek, B.R. Bułka, A. Fert, Phys. Rev. B 62, 12363 (2000).

[17] J. Martinek, J. Barnaś, G. Schön, S. Takahashi, S. Maekawa, J. Supercond. 16, 343 (2003).

[18] T. Fujisawa, D.G. Austing, Y. Tokura, Y. Hirayama, S. Tarucha, Phys. Rev. Lett. 88, 236802 (2002).

[19] L.I. Glazman, M.E. Raikh, JETP Lett. 47, 452 (1988); T.K. Ng, P.A. Lee, Phys. Rev. Lett. 61, 1768 (1988).

[20] S. Sasaki, S. De Franceschi, J.M. Elzerman, W.G. van der Wiel, M. Eto, S. Tarucha, L.P. Kouvenhoven, Nature (London) 405, 764 (2000); J. Gores, D. Goldhaber-Gordon, S. Heemeyer, M.A. Kastner, H. Shtrikman, D. Mahalu, U. Meirav, Phys. Rev. B 62, 2188 (2000).

[21] N. Sergueev, Q.F. Sun, H. Guo, B.G. Wang, J. Wang, Phys. Rev. B 65, 165303 (2002).

[22] J. Martinek, Y. Utsumi, H. Imamura, J. Barnaś, S. Maekawa, J. König, G. Schön, cond-mat/0210006 (2002).

[23] R. Świrkowicz, J. Barnaś, M. Wilczyński, to be published.

[24] D. Weinmann, W. Häusler, B. Kramer, Phys. Rev. Lett. 74, 984 (1995); H. Akera, Phys. Rev. B 60, 10683 (1999).

[25] K. Tsukagoshi, B.W. Alphenaar, H. Ago, Nature (London) 401, 572 (1999).

[26] L.I. Glazman, K.A. Matveev, Pis'ma Zh. Eksp. Teor. Fiz. 48, 403 (1988) [JETP Lett. 48, 445 (1988)].

[27] W. Rudziński, J. Barnaś, Phys. Rev. B 64, 085318 (2001).

[28] H. Schoeller, G. Schön, Phys. Rev. B 50, 18436 (1994); J. König, H. Schoeller, G. Schön, Phys. Rev. B 58, 7882 (1998).

[29] T.A. Costi, Phys. Rev. Lett. 85, 1504 (2000); J.E. Moore, X.-G. Wen, Phys. Rev. Lett. 85, 1722 (2000).

[30] G.A. Prinz, J. Magn. Magn. Mater. 200, 57 (1999).

[31] R. Świrkowicz, J. Barnaś, M. Wilczyński, J. Phys., Condens. Matter 14, 2011 (2002).

[32] A.P. Jauho, N.S. Wingreen, Y. Meir, Phys. Rev. B 50, 5528 (1994). 